$\underset{\text { clinical }}{\text { nephron }}$

Practice
Nephron 2019;143:174-178

DOI: 10.1159/000500167
Received: March 12, 2019

Accepted after revision: April 4, 2019 Published online: April 24, 2019

\title{
Sepsis-Associated Acute Kidney Injury: A Problem Deserving of New Solutions
}

\author{
John A. Kellum ${ }^{a, b}$ Xiaoyan Wen ${ }^{a, b}$ Mark P. de Caestecker ${ }^{c}$ Neil A. Hukriede ${ }^{a, d}$

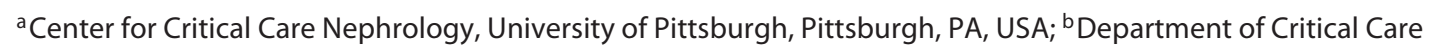 \\ Medicine, University of Pittsburgh, Pittsburgh, PA, USA; ${ }^{C}$ Department of Medicine, Vanderbilt University Medical \\ Center, Nashville, TN, USA; ${ }^{d}$ Department of Developmental Biology, University of Pittsburgh, Pittsburgh, PA, USA
}

\section{Keywords}

Embryonic kidney progenitor cell · Renal repair ·

Sepsis-associated acute kidney injury

\section{Abstract}

Sepsis-associated acute kidney injury (S-AKI) significantly worsens patient prognosis, and recent evidence suggests that the injury process begins early and may be sustained by therapies used to treat the sepsis (e.g., fluids resuscitation, antibiotics). While efforts to develop less-injurious treatments are making progress, some degree of secondary injury is to be expected. So too is the inevitable nature of organ injury, which is often present at the time the patient seeks medical attention. We recently found that most patients presenting with septic shock and developing AKI had evidence of kidney damage at the time of, or within $24 \mathrm{~h}$ of their admission. In such patients, prevention is not a viable option,
\end{abstract}

as injury has already occurred by the time of presentation. Since S-AKI patients are at increased risk of developing chronic kidney disease, a fundamental target for interventions in S-AKI is to prevent fibrosis (maladaptive repair) while stimulating regeneration (proliferation of viable epithelial cells). Using a pathway-agnostic, proliferation-based phenotypic assay, we discovered phenylthiobutanoic acid, a small molecule histone deacetylase inhibitor, that enhances renal recovery and reduces fibrosis in both zebrafish and mouse models of AKI.

(c) 2019 S. Karger AG, Basel

Contribution from the AKI and CRRT 2019 Symposium at the 24th International Conference on Advances in Critical Care Nephrology, Manchester Grand Hyatt, San Diego, CA, USA, February 26 - March 1, 2019. This symposium was supported in part by the NIDDK funded University of Alabama at Birmingham-University of California San Diego O'Brien Center for Acute Kidney Injury Research (P30DK079337).

\section{KARGER}

() 2019 S. Karger AG, Basel

E-Mail karger@karger.com

www.karger.com/nef
John A. Kellum, MD, MCCM

Center for Critical Care Nephrology

University of Pittsburgh

3347 Forbes Avenue, Suite 220, Pittsburgh, PA 15213 (USA)

E-Mail kellumja@upmc.edu 


\section{Introduction}

Sepsis-associated acute kidney injury (S-AKI) is a major public health problem. At least $10 \%$ of hospitalized patients and more than $50 \%$ of patients admitted to ICU have or will develop AKI with older individuals are disproportionately affected [1]. This same demographic relationship also exists for sepsis, the leading cause of AKI. Recent evidence also suggests that much milder forms of AKI are associated with increased risk of hospital mortality. Although the reasons for this increased mortality are not fully understood, these studies and many others make a compelling argument that patients who develop AKI are at an additional increased risk of death that is in some way due to AKI itself. S-AKI is usually already present when patients seek medical attention. In a recent study of 1,243 patients with septic shock, we found that 859 (69.1\%) developed AKI by KDIGO criteria [2]. Among these patients, the majority, 626 (72.9\%), already had clinical evidence of AKI at enrollment and of the remaining patients, two thirds manifested AKI within $24 \mathrm{~h}$ suggesting that injury had already occurred prior to admission. Thus, for S-AKI, therapies will need to be effective hours to days after injury has occurred.

\section{New Therapies for S-AKI are Urgently Needed}

Over the last 30 years, attempts to improve outcomes for patients with AKI have targeted hemodynamics, diuretics (including natriuretic peptides), and oxidative stress. All have been spectacular failures [3]. Nearly 15 years ago, Early Goal-Directed Therapy (EGDT) was first described and rapidly became a standard for management of septic shock [4]. In addition to reduced 28-day mortality as compared to control patients (49.2 vs. $33.3 \%, p=0.01$, patients randomized to EGDT expressed lower concentrations of proinflammatory cytokines and lower circulating levels of caspase-3 compared to control patients. Based on these data, EGDT should not only improve survival but should also decrease the incidence of AKI. However, recent multicenter trials of EGDT failed to show improved survival [5]. Furthermore, we observed no reduction in AKI or inflammation. The lack of effect of EGDT likely relates to changes in background care including early resuscitation and antibiotics. Still, while hospital survival has improved, AKI occurred in $69 \%$ of patients and longer-term survival remains poor (1-year mortality exceeded $40 \%$ [6]). Many promising new therapies are unlikely to benefit S-AKI

S-AKI: A Problem Deserving of New Solutions because damage has usually already occurred at the time of presentation.

Failure to develop effective therapies for AKI has occurred, in part, because patients often present late in the course of AKI. Experimental drugs found to be ineffective in clinical trials for AKI were only effective in experimental models of AKI when given before the initiating injury. In addition, failure to model injury on backgrounds of aging, diabetes, and CKD may account for the failure of preclinical practice, thereby translating into clinical practice [7]. Finally, access to biopsy samples from injured human kidney that can be used to correlate pathology with changes at the molecular level is severely limited, thus not allowing for a traditional target-based drug discovery strategy [8]. To overcome these limitations, we developed a pathway-agnostic, proliferationbased phenotypic assay that measures the effects that mitigate maladaptive repair associated with persistent cell cycle arrest, a cellular phenomenon reflective of AKI in both animal models and patients [9]. Using this approach, we discovered phenylthiobutanoic acid (PTBA), a small molecule that enhances recovery and reduces fibrosis, when administered after injury in both zebrafish and mouse AKI. To deliver PTBA, we developed the prodrugs UPHD25 (methyl ester) and UPHD186 (benzamide) [9-11].

\section{Identification of PTBA}

Renal repair occurs largely by the proliferation of surviving tubular epithelial cells a number of days after the initiating injury. Therefore, we developed a high content phenotypic screen using zebrafish embryos to identify compounds that promote the proliferation of embryonic kidney progenitor cells (EKPCs) [9-11]. Since proliferating tubular epithelial cells express markers of kidney progenitor cells after injury, we reasoned that compounds promoting expansion of zebrafish EKPCs might also increase the proliferation of epithelial cells after AKI. Using this approach, we identified PTBA, which expands EKPCs in zebrafish embryos [9, 11]. In addition to PTBA $\left(\mathrm{EC}_{50}=1.48 \mu \mathrm{M}\right)$, we evaluated several ester and benzamide prodrugs: UPHD25 $\left(\mathrm{EC}_{50}=0.65 \mu \mathrm{M}\right), \operatorname{UPHD} 36\left(\mathrm{EC}_{50}=1.41 \mu \mathrm{M}\right)$, and UPHD186 $\left(\mathrm{EC}_{50}=0.77 \mu \mathrm{M}\right)[9-11]$. While the evaluation of PTBA prodrugs using EKPC assays indicates these assays have predictive value for efficacy when moving into mouse AKI models [12], initiating a progression to a large number of compounds from EKPC 


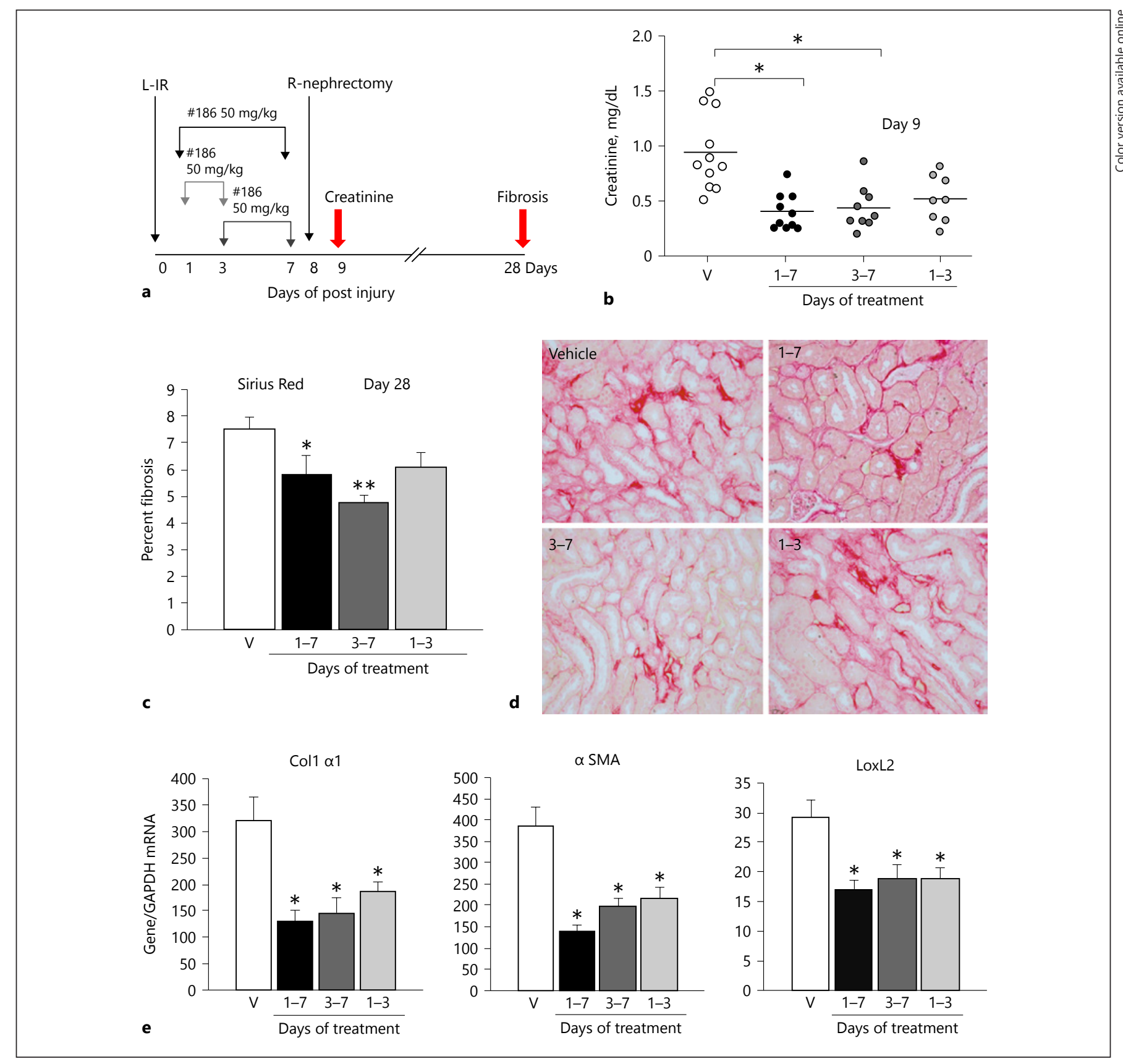

Fig. 1. Delayed treatment with UPHD186 reduces fibrosis after IRAKI. UPHD186 at $50 \mathrm{mg} / \mathrm{kg} /$ day days $1-7,1-3$ and 3-7, as indicated. a Model; (b) serum creatinine at day 9; (c) percent fibrosis; (d) sirius red stained kidneys. e Fibrosis marker mRNAs 28 days. ANOVA, Bonferroni, ${ }^{*} p<0.05,{ }^{* *} p<0.005$ vs. vehicle. Adapted from [10] (with permission). assays directly to mouse AKI screens is impractical. We therefore developed an assay that distinguishes compounds using a more stringent model. Gentamicin-induced AKI (referred to as zfAKI) in zebrafish larvae [13] recapitulates injury and proliferation-based repair in mammalian AKI but allows for higher throughput anal- ysis of compound efficacy in AKI than mouse models $[12,13]$. As fish are soaked in compounds, this assay is not subject to drug delivery and absorption problems that can occur when unoptimized compounds are used in vivo. To determine whether the zfAKI model is predictive of efficacy in mice, we evaluated whether a vari- 
ety of EKPC positive compounds improve larval survival after gentamicin-induced AKI [10]. PTBA and UPHD25, 36, and 186 had beneficial effects on larval survival, with PTBA, UPHD25 and 186 showing the most benefit [10]. However, another compound, UPHD29, which is active in the EKPC assay, had no effect on post-AKI survival. Interestingly, when translating this work to a mouse model of AKI (see below), we found that both prodrugs, UPHD25 and 186, delivering PTBA, were active, while UPHD29 was inactive [10]. These data established zfAKI as a useful secondary screen predictive of efficacy in mouse AKI; eliminated a positive compound from the EKPC primary screen (UPHD29) that was inactive in mice; and allowed us to efficiently prioritize compounds before advancing to rodent efficacy studies.

The prodrugs UPHD25 and UPHD186 reduce fibrosis in ischemic, toxic, and obstructive models of AKI when administered for short periods (days) after the initial insult $[10,12]$, with UPHD186 even showing efficacy when administration is delayed 3 days after severe ischemia reperfusion IR-AKI in mice (Fig. 1) [10]. We have also shown that only UPHD186 is effective in reducing fibrosis after unilateral ureteral obstruction [10] and that it has no identifiable toxicity at $5 \times$ the effective dose (not shown).

\section{The Challenge of Post S-AKI Treatment Strategy}

$\mathrm{S}$-AKI is a challenging clinical problem with long-term consequences. Most strategies for S-AKI have focused on kidney "resuscitation" or on protecting the kidney from systemic inflammation. Our strategy focuses on assisting in recovery. For the past 6 years, we have conducted a multicenter study enrolling patients with sepsis-associated AKI and conducting long-term follow-up. In this study, we have advised patients to undergo biomarker testing in the acute phase and to obtain in-home visits at $3,6,12,24$, and 36 months for physical exams, medication review, quality of life assessment, and blood/urine sampling. Our early results show that patients who recover renal function by hospital discharge have 1-year survival equivalent to those without AKI at all, while non-recovery patients have extremely poor outcomes that are largely determined by day 30 [2]. Thus, targeting renal recovery after sepsis has significant appeal.

PTBA has activity as a deacetylase inhibitor of histone [11] and other non-histone proteins. Renal histone H4 hyper-acetylation was used as a readout of target engagement for pre-clinical dosing with PTBA analogs in mice and proved superior to traditional HDAC inhibitors including MS-275 and SAHA [10, 12]. In our hands, MS-275 is inactive in the zfEKPC proliferation assay [10]. Moreover, while SAHA, UPHD25, and 186 increase tubular proliferation in zebrafish and mouse AKI, MS275 inhibits proliferation of cultured tubular epithelial cells [14]. Thus, UPHD186 appears to be the most promising candidate for a pro-proliferative, anti-fibrotic agent that can be administered post-injury. We are actively studying the effect of this compound in other AKI models including sepsis.

\section{Disclosure Statement}

N.A.H. discloses a patent USP 9670236, on UPHD186 held by the University of Pittsburgh. No other authors declared any competing interests.

\section{Funding Sources}

Supported by National Institutes of Health (NIH)/National Institute of Diabetes and Digestive and Kidney Diseases grants R01DK083961, 2R01DK069403, 1R01DK112652, R01DK112688, and 1P30DK079307; U.S. Department of Defense W81XWH-17-1-0610.

\section{References}

1 Collins AJ, Foley RN, Chavers B, Gilbertson D, Herzog C, Ishani A, et al. US Renal Data System 2013 Annual Data Report. Am J Kidney Dis. 2014 Jan;63(1 Suppl):A7-7.

2 Kellum JA, Chawla LS, Keener C, Singbartl K, Palevsky PM, Pike FL, et al.; ProCESS and ProGReSS-AKI Investigators. The Effects of Alternative Resuscitation Strategies on Acute Kidney Injury in Patients with Septic Shock. Am J Respir Crit Care Med. 2016 Feb;193(3): $281-7$.
3 Winkelmayer WC, Finkel KW. Prevention of acute kidney injury using vasoactive or antiplatelet treatment: three strikes and out? JAMA. 2014 Dec;312(21): 2221-2.

4 Rivers E, Nguyen B, Havstad S, Ressler J, Muzzin A, Knoblich B, et al.; Early Goal-Directed Therapy Collaborative Group. Early goal-directed therapy in the treatment of severe sepsis and septic shock. N Engl J Med. 2001 Nov;345(19):1368-77.
5 Angus DC, Barnato AE, Bell D, Bellomo R, Chong CR, Coats TJ, et al. A systematic review and meta-analysis of early goal-directed therapy for septic shock: the ARISE, ProCESS and ProMISe Investigators. Intensive Care Med. 2015 Sep;41(9):1549-60.

6 Yealy DM, Kellum JA, Huang DT, Barnato AE, Weissfeld LA, Pike F, et al.; ProCESS Investigators. A randomized trial of protocolbased care for early septic shock. N Engl J Med. 2014 May;370(18):1683-93.
S-AKI: A Problem Deserving of New Solutions
Nephron 2019;143:174-178 DOI: $10.1159 / 000500167$ 
7 Skrypnyk NI, Siskind LJ, Faubel S, de Caestecker MP. Bridging translation for acute kidney injury with better preclinical modeling of human disease. Am J Physiol Renal Physiol. 2016 May 15;310(10):F972-84.

8 de Caestecker M, Harris R. Translating Knowledge Into Therapy for Acute Kidney Injury. Semin Nephrol. 2018 Jan;38(1):88-97.

9 Sanker S, Cirio MC, Vollmer LL, Goldberg ND, McDermott LA, Hukriede NA, et al. Development of high-content assays for kidney progenitor cell expansion in transgenic zebrafish. J Biomol Screen. 2013 Dec;18(10):1193-202.
10 Skrypnyk NI, Sanker S, Skvarca LB, Novitskaya T, Woods C, Chiba T, et al. Delayed treatment with PTBA analogs reduces postinjury renal fibrosis after kidney injury. Am J Physiol Renal Physiol. 2016 Apr;310(8):F70516.

11 de Groh ED, Swanhart LM, Cosentino CC, Jackson RL, Dai W, Kitchens CA, et al. Inhibition of histone deacetylase expands the renal progenitor cell population. J Am Soc Nephrol. 2010 May;21(5):794-802.

12 Cianciolo Cosentino C, Skrypnyk NI, Brilli LL, Chiba T, Novitskaya T, Woods C, et al. Histone deacetylase inhibitor enhances recovery after AKI.JAmSocNephrol.2013May;24(6):943-53.

13 Cianciolo Cosentino C, Roman BL, Drummond IA, Hukriede NA. Intravenous microinjections of zebrafish larvae to study acute kidney injury. J Vis Exp. 2010 Aug;(42): 2079.

14 Liu N, He S, Ma L, Ponnusamy M, Tang J, Tolbert E, et al. Blocking the class I histone deacetylase ameliorates renal fibrosis and inhibits renal fibroblast activation via modulating TGF-beta and EGFR signaling. PLoS One. 2013;8(1):e54001. 